and positions of all the nebulæ known at the end of 1887, was published in 1888 (Mem. Roy. Astro. Soc., 49, Part 1; 1888), and this was later supplemented by his "Index Catalogue of Nebulæ found in the Years 1888 to 1894 , with Notes and Corrections to the New General Catalogue" (Mem. 51; 1895) and by the "Second General Catalogue" (Mem. 59; 1908), which provided similar data up to the end of 1894. The Royal Astronomical Society decided to reproduce the three sections by photographic means and to include them in one volume under the title "Memoirs of the Royal Astronomical Society. New General Catalogue of Nebulæ and Clusters of Stars (1888). Index Catalogue (1895). Second Index Catalogue (1908)" (from the Society, Burlington House, London, W.1; 1953 ; $£ 310 s$. post free, or $£ 210 s$. to Fellows). This is very much more convenient for observers than the three separate volumes and, except for the alteration in the pagination of the two Index Catalogues to follow on from that of the "New General Catalogue", the catalogue has remained unaltered. In its present form this volume should prove very acceptable to observers of nebulæ and clusters.

\section{Design and Accuracy of Volumetric Glassware}

THE sixth in the series of "Notes on Applied Science" prepared by the National Physical Laboratory, Teddington, is entitled "Volumetric Glass. ware: Scientific Aspects of Design and Accuracy" (pp. 22. London: H.M.S.O., 1954; 1s. 6d. net). It presents the basic scientific information which must be taken into account in the preparation of standards for volumetric glassware and sets out the main features of standardization which are of general applicability in rationalization or verification. The information given is supplementary to that of any test pamphlets issued by the National Physical Laboratory and also to the British Standards issued by the British Standards Institution. The booklet does not discuss techniques of manufacture or the uses to which the glassware is put. It consists of three main sections dealing with the basis of graduation, the construction and verification of apparatus, and the dependence of volume delivered on delivery procedure. The first section contains a clear explana. tion of the three units of volume in use-the litre, imperial gallon and cubic foot-and the relationship between them; a discussion of the effect of tem. perature on the capacity of a glass vessel ; and the definition of capacity involving either a volume of water or mercury and, in the case of a graduated glass vessel, the precise setting of the liquid meniscus to a horizontal line. Various details of procedure to be adhered to in the cleaning and in the determination of the capacity of a glass vessel are included in the second section. It is emphasized that all traces of detergents, which are now being increasingly used for cleaning glassware, must be carefully removed from volumetric glassware before calibration, otherwise the surface tension of the water used in the calibration, and consequently the shape of the meniscus, will be considerably affected. Also, all determinations of a glass vessel are based ultimately on the gravimetric method, so that when water is used for calibration and high accuracy is required, both the temperature and pressure of the air at the time of weighing should be recorded. The final section contains a detailed discussion of delivery times and drainage times of burettes and pipettes and is illustrated by several graphs and tables.
The booklet concludes with a list of references and of British Standards relating to volumetric glassware.

\section{Institution of Gas Engineers: Annual Report}

THE ninety-first annual report and accounts of the Council of the Institution of Gas Engineers (Pub. No. 440 ; pp. 42 ; 'from the Institution, London; 1954), presented at the annual general meeting of the Institution in Bournemouth during May 31June 2, includes a list of communications during the year, and the regulations for the award of the Charles Hunt Memorial Medals in gas engineering are ap. pended. Five supplements to the fourteenth report of the Chairman's Technical Committee were issued during the year by the Gas Works Safety Rules Committee. These were concerned with a model specification for platforms, ladders and stairways to provide means of access to plant, a code of practice for the safe operation of tower purifiers, a note on the operation of electro-detarrers with carburetted water-gas plant, safety recommendations for electrical equipment in gas manufacture, purification and distribution, and additional references to the bibliography of literature relating to accident prevention. A booklet on "Unusual Incidents at Gasworks" was due to be published, and a supplement dealing with the internal protection of gas pipes has been issued by the Pipes Committee.

\section{Museum of Comparative Zoology at Harvard} College : Annual Report for 1952-53

The annual report for 1952-53 of the Museum of Comparative Zoology at Harvard College (pp. 24; from the Museum, Cambridge, Mass., 1953) details the work of an institution which, though burdened with an antiquated name, executes an extremely important task. It is in effect mainly a research institution in which function is deemed of more importance than taxonomy, though studies in the latter are still undertaken. In addition, important additions have been made to the Museum collections, and several expeditions, including a survey of molluscs at the site of the Jim Woodruff Dam at Chattahoochee, Florida, have been undertaken. An impressive list of ninety-five papers published by the staff is some indication of the continued activity of this well-known Museum.

\section{Reactor School at Harwell}

As a step towards encouraging industry to play a greater part in the development of atomic power, the United Kingdom Atomic Energy Authority has opened a reactor school at Harwell. The new school will provide, for a fee of $£ 250$, a three-months course of training for staff from industrial concerns to learn the techniques by which heat from atomic piles can be converted into useful power. Priority will be given at first to staff of firms already acting as contractors to the Atomic Energy Authority. Some new graduate members of the Authority's own staff will also be trained in the school. Three courses will be held each year, the next in January 1955. The syllabus includes lectures and individual instruction in nuclear physics, reactor physics, metallurgy and reactor engineering, as well as experimental work. Lectures are given by experts drawn from various divisions at Harwell, and by the full-time school staff. Applications for places in the school should be made to the Manager, Reactor School, A.E.R.E., Harwell, Berkshire, and should give sufficient 